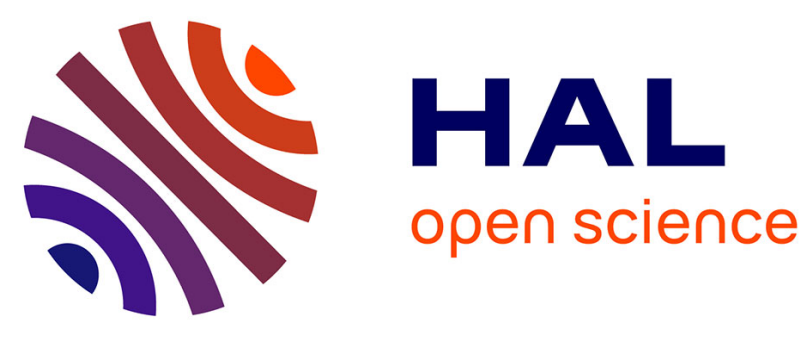

\title{
Ce voile qui cache la forêt
}

Houria Abdelouahed

\section{To cite this version:}

Houria Abdelouahed. Ce voile qui cache la forêt. Topique - Revue freudienne, 2010, La psychanalyse au Maghreb et au Machrek, 1 (110), pp.183 - 195. 10.3917/top.110.0183 . hal-01508600

\section{HAL Id: hal-01508600 https://hal.science/hal-01508600}

Submitted on 16 Jun 2017

HAL is a multi-disciplinary open access archive for the deposit and dissemination of scientific research documents, whether they are published or not. The documents may come from teaching and research institutions in France or abroad, or from public or private research centers.
L'archive ouverte pluridisciplinaire HAL, est destinée au dépôt et à la diffusion de documents scientifiques de niveau recherche, publiés ou non, émanant des établissements d'enseignement et de recherche français ou étrangers, des laboratoires publics ou privés. 


\title{
Ce voile qui cache la forêt
}

\author{
Houria Abdelouahed
}

Ne t'étonne pas s'il baise tes cheveux bouclés ;

Que peut faire un fou, sinon mordre ses chaînes?

Rûmî, Rubâ'iyyât

Devant les mains frêles d'Antigone s'acharnant à enterrer son frère, JeanPierre Vernant écrit : «Celui qui a consenti à porter un nom et qui a possédé un visage, mérite l'acte des funérailles.»

Nom et visage se trouvent ainsi enlacés au plus profond de la dimension symbolique. Et Jean-Pierre Vernant d'ajouter : «Le nom et le visage est ce qui constitue l'identité d'une personne. » Si le nom adosse l'humain à sa condition d'être mortel, le visage, parce que voyant visible, est ce qui permet à l'homme de s'appréhender dans l'œil de son vis-à-vis, de voir le reflet dédoublé de luimême. L'étymologie latine (vultus, us) ou grecque (prosapon) ou arabe (wajh, de la même racine que la muwâjaha, le face à face) rappelle cet « entrelacs » (M. Merleau-Ponty) entre voir et être vu. C'est dans le face-à-face que l'homme peut se regarder. Toutefois, «ce qu'on présente de soi au regardd'autrui et qui est le socle de mon identité ${ }^{1} \gg$ est accolé à la nomination. Ainsi, l'assomption de l'identité est déjà une assomption de l'altérité parce que le champ de la parole et du langage permet «la métaphorisation de l'aliénation ${ }^{2}$ comme pivot et fondement de l'organisation symbolique.

1. J.-P. Vernant, L'individu, la mort, l'amour, Paris, Gallimard, 1989, p. 118.

2. Expression de P. Legendre, Dieu au miroir, Fayard, 1994, p. 56. C'est l'un des enjeux de la nouvelle de Stéphan Zweig, Le joueur d'échecs, où la possibilité de se mirer dans le regard d'autrui lui est refusé. Il dira: «Pas d'autre visage que le mien», Le joueur d'échecs, Stock, 1990. 
Or, le nouveau malaise dans la culture arabo-musulmane, à travers le problème du hijâb (traduit par «voile») pivote autour de cet entrelacs entre voir et être vu. Défaire la réflexivité entre le voyant et le visible, de telle sorte que le voyant (la femme) cesse d'être visible, tel est le malaise auquel nous confronte le problème du voile mobilisant ainsi une multiplicité de champs d'intervention et de discours : théologiques, sociaux, psychanalytiques, politiques à travers la question de la laïcité, juridique...

\section{L’ÉPAISSEUR D’UNE CONFUSION}

Le vocable al-hijâb (qu'on traduit par voile) n'est nullement mentionné par le Coran comme synonyme d'un quelconque vêtement féminin. Il figure comme métaphore, « nos cœurs sont enveloppés d'un voile épais » (Cor. 38 $: 32 ; 41: 5 ; 17: 45$ ), comme ligne séparant le paradis de la Géhenne ${ }^{3}$ laquelle ligne relève plutôt de ce qu'Ibn Arabi nomme 'âlam al-khayâl, ou monde imaginal, dans la traduction de Henry Corbin -, dans l'opposition humain/divin, comme le signe d'une altérité radicale ${ }^{4}$ et enfin, pour désigner la vision interdite du divin dans l'au-delà (c'est l'adjectif mahjûbûn, voilés, qui est utilisé). ${ }^{5}$

Dans son acception matérielle, le hijâb figure deux fois comme l'équivalent d'un rideau. Dans la sourate 19, il vient séparer Marie, dans son recueillement, des siens ${ }^{6}$ et dans la sourate 33 , pour distinguer les femmes du prophète: «Quand vous demanderez quelque objet aux épouses du Prophète, faites-le derrière un voile (hijâb). Cela est plus pur pour vos cœurs et pour leurs cœurs » (Cor.33-53).

Dans la seconde sourate, le hijâb n'est plus le signe de la spiritualité (ici de la femme qui transcende le terrestre au nom de l'amour divin), mais vient désigner l'espace privé du prophète. Le grand commentateur et exégète Tabarî (IX ${ }^{\mathrm{e}}$ siècle) donne l'interprétation suivante: «Afin de célébrer son mariage avec Zaïnab, réputée pour sa grande beauté, le prophète invita quelques amis. Mais ces derniers, après le repas, tardèrent à partir empêchant le prophète de jouir de la nouvelle épouse. Las, ce dernier dut attendre, néanmoins, le départ du dernier convive avant d'énoncer avec fermeté : «C'est un hijâb (rideau) entre vous et moi ». Le vocable, ici, est en lien intime avec la puissance du désir et la sexualité. Une autre version relate un incident survenu lors d'un

3. «Un voile épais est placé entre le paradis et la géhenne. » (Cor. 7: 46)

4. «Un voile est placé entre nous et Toi » (Cor. $41: 5)$

5 . « Non, ils seront ce jour-là voilés de leur Seigneur» (Cor. $83: 15)$. Denise Masson traduit: «séparés de leur Seigneur».

6. «Elle plaça un voile entre elle et les siens » (Cor. $19: 17$ ). 
repas où la main d'un convive frôla celle d'Aïcha, la jeune épouse du prophète. Dans les deux versions, ce sont les épouses de ce dernier qui font l'objet de l'intérêt coranique qui rappelle : « Ô, vous les femmes du prophète ! Vous n'êtes comparables à aucune autre femme ». Et le Coran de continuer : «Restez dans vos maisons, ne vous montrez pas dans vos atours comme le faisaient les femmes au temps de l'ancienne ignorance (jâhiliyya ûlâ) »' Femmes, explique Tabari, devant se souscrire à toute tentation afin de ne pas attiser le désir de l'adultère. D'où cette injonction qui fut traduite par : « $\mathrm{Ne}$ vous montrez pas dans vos atours (walâ tabarrajna) comme le faisaient les femmes au temps de l'ancienne ignorance. » (traduction de Denise Masson), «Demeurez dans vos maisons. Ne vous parez pas des parures de l'ignorance originelle» (trad. André Chouraqui), «Gardez-vous dans vos maisons, abstenez-vous des exhibitions du premier paganisme» (trad. Jacques Berque).

Le terme de tabarruj (d'où tabarrajna, traduit par : ne vous exhibez pas, abstenez-vous, ne vous montrez pas dans vos parures, ne vous parez pas des parures...) est, dans les différentes traductions, pris comme synonyme d'un accoutrement compromettant car trop séducteur, désigne en fait tabakhtur, à savoir la démarche. Quant à l'expression « la première jâhiliyya (paganisme, première ignorance...», elle s'offre, dans le flux des interprétations de Tabarî, comme un temps insaisissable, temps qui se perd dans les brumes d'un passé aux traces indiscernables, aux contours plus mythiques qu'historiques.

Or, les théologiens d'aujourd'hui relient directement ce verset qui s'adresse exclusivement aux épouses du prophète à un autre demandant aux croyantes « de rabattre leurs voiles sur leurs poitrines, de ne montrer leurs atours qu'à leurs époux ou à leurs pères...» (Cor. $24: 31$, traduction de Denise Masson) ${ }^{8}$. Or, le Texte demande de rabattre le khimâr (le voile) sur les juyûb, (les fentes). S'agit-il des fentes sexuelle et fessière (d'où l'utilisation du pluriel), ou seulement de l'ouverture entre les deux seins ? Et que désigne 1'injonction de «ne montrer que l'extérieur de leur beauté » ? S'agit-il de la beauté du corps, celle des artifices féminins, ou encore de la beauté du vêtement? Ce dernier, comme le rappelle le grand théologien Râzî (XIII' siècle) qui conseille, d'ailleurs, à la femme de s'habiller en fonction des conditions du travail et du contexte géographique, peut être un artifice apprécié, comme en témoigne la sourate $7 .{ }^{9}$

7. Cor. $33: 32-33$.

8. « de ne pas faire montre de leurs agréments, sauf ce qui en émerge, de rabattre leur fichu sur les échancrures de leur vêtement » (trad. J. Berque) ; « de ne montrer que l'extérieur de leur beauté, de rabattre leur voile sur leur gorge » (trad. André Chouraqui) ; « de ne laisser paraître de leur charme que ce qu'elle ne peuvent dissimuler, de couvrir leur gorge d'un voile, de ne laisser voir les parties découvertes » (trad. O. Pesle et A.Tidjani).

9. Cor. 7: 31 . 


\section{COMPLEXITÉS}

Si l'on fait travailler le Texte, on se heurte rapidement à des contradictions incontournables, voire à des impasses. Les exégètes stipulent que le verset (24:31) ne vise que les femmes libres, et non les jawârî (les femmes esclaves) qui, parce qu'elles sont objet de vente et d'achat, dit Râzî, nécessitent le regard et l'approche palpable du corps. La femme esclave est dans l'obligation de dévoiler ce que la femme libre est appelée à dissimuler. L'habit sert également à distinguer la musulmane de la dhumia, celle qui doit payer une taxe pour pouvoir conserver sa religion. Ainsi, le voile devient un signe de discrimination. Suivant cette logique, l'habit fait le moine. " Dis-moi comment tu t'habilles, je te dis si tu es libre ou esclave, musulmane ou mécréante ». Et si l'on accepte le verset dans son acception première, celle des fuqahâ' (les docteurs de la loi), on doit accepter l'esclavage et la séquestration des femmes dites libres dans un espace clos où tout leur être ne s'accomplit que dans la maternité et les activités domestiques.

La suite du verset est encore plus problématique : «...de ne montrer leurs atours qu'à leurs époux, ou à leurs pères (...) ou à leurs servantes ou leurs esclaves ou à leurs serviteurs mâles incapables d'actes sexuels, ou aux garçons impubères $\gg^{\mathbf{1 0}}$. Au-delà du fait que les données cliniques et anthropologiques nous montrent que le corps peut être source d'excitation pour un garçon impubère ${ }^{11}$ et au-delà de l'asymétrie qui est bien visible (l'homme peut jouir de la femme servante désignée comme mâ malakat al-yamîn, ce qu'il possède, tandis que la femme ne peut jouir de l'homme esclave), ce qui est frappant c'est que ce dernier, est présenté dans les commentaires des exégètes, comme dépourvu de sentiments, d'affects, d'émotions, de désir ou tout simplement d'humanité. C'est comme si sa condition d'homme esclave avait pour conséquence une forclusion de la capacité affective et subjective. Nos exégètes ignorent-ils que c'est l'interdit qui crée le désir? Méconnaissent-ils à ce point l'Histoire et la poésie préislamique où la figure d'Antara ${ }^{12}$, l'esclave noir, transcenda, dans l'amour de la femme interdite (blanche et libre), sa condition sociale dans une Arabie esclavagiste préislamique? Comment se fait-il que

10. Cor. 24 :33. « Elles ne laisseront voir leurs agréments qu'à leur mari, à leurs enfants, à leur pères, beaux-pères, fils, beaux-fils, frères, neveux de frères ou de sœurs, aux femmes (de leur communauté), à leurs captives, à leurs dépendants hommes incapables de l'acte, ou garçons encore ignorants de l'intimité des femmes. » (trad. J. Berque); «Elles ne montreront leur beauté qu'à leurs époux, ou à leurs fils, ou aux fils de leurs époux (...) ou aux esclaves exempts de convoitise parmi les hommes, ou aux garçons qui ne sont pas attirés par le giron des femmes », (trad. A. Chouraqui).

11. Freud nous a montré comment la pulsion sexuelle s'étaie sur la pulsion d'autoconservation.

12. Cf. Le Dîwân de la poésie arabe classique (trad. Abdelouahed H. \& Adonis), Gallimard /Poésie, 2008. 
cette capacité humaine d'être désirant fut déniée à un esclave après l'avènement de l'islam, alors que transcender les conditions d'avilissement et d'esclavage était permis ou du moins possible pendant la période d'al-jâhiliyya, considérée pourtant comme temps de paganisme, d'obscurantisme et d'ignorance d'avant la civilisation?

Ces contradictions persistent jusqu'à aujourd'hui comme en témoigne l'immense succès d'un Qaradawî, considéré comme un savant moderne éclairé et dont les écrits «L'habit de la notoriété et de la vantardise, la taille des sourcils pour les amincir...» $)^{13}$ n'ont rien à envier à Tertullien ou à Saint Paul écrivant que « la femme écoute l'instruction en silence avec une entière soumission ». Dans le chapitre qui s'intitule « la sage raison de permettre la polygamie», Qaradawi explique que l'islam, qui est la parole ultime de Dieu avec laquelle Il clôtura les missions, apporta une législation universelle. Or, comme «il y a parmi les gens celui qui désire ardemment procréer, mais qui a eu pour épouse une femme frigide ou malade, ou ayant des règles trop prolongées ou autre anomalie, tandis que l'homme ne peut supporter longtemps la privation de femmes. N'a-t-il pas le droit, dans ce cas, d'épouser une autre femme ?...». On peut rétorquer: «Si la sexualité n'a pour finalité que la procréation, pourquoi avoir une autre épouse en cas de frigidité de la femme, puisque frigidité n'est pas incompatible avec maternité ?» Mais «c'est là qu'il est de l'intérêt de la société et des femmes elles-mêmes que les femmes soient co-épouses plutôt que de rester toute leur vie de vieilles demoiselles privées de la vie conjugale», conclut-il.

Accepter la polygamie, sinon la jeune fille risque de rester vieille demoiselle et la femme de se voir mutallaqa ou tâliq ${ }^{14}$. Le vocable (mutallaqa ou tâliq) fait partie du vocabulaire bestiaire. Il se rapporte à une chamelle, débarrassée de ses entraves, qu'on envoie au pâturage brouter à sa guise. L'image, de par cette référence au bestiaire et au broutage, fait penser à l'organisation la plus primitive de la sexualité infantile : la phase orale. Ainsi, la mutallaqa est dans le pulsionnel primitif qui dévaste tout sur son passage en l'absence... d'un surmoi.

La burqa qui revient de façon récurrente dans le débat sur le voile appartient également au vocabulaire bestiaire. En arabe al-burqu' ou al-burqa' est un terme «fort connu, il est réservé aux dawâb et aux femmes des bédouins » nous dit Ibn Manzûr ${ }^{15}$. Or, dawâb (pluriel de dâbba) désigne les montures, les animaux rampants, ou les bêtes de somme. Le vocable désigne également le

13. Youssef Qaradawi, Le licite et l'illicite en islam., réédition Dâr - al qalam, en 2004.

14. La forme grammaticale implique, jusqu'à aujourd'hui, qu'elle est celle qui subit l'action même si c'est elle qui demande le divorce. La forme passive signifie qu'elle n'est pas le sujet.

15. Ibn Manzûr, Lisân al- 'arab (encyclopédie philologique du XIII' ${ }^{\mathrm{c}}$ siècle), tome 1, p. 264-265. 
chanfrein du cheval et ce que mettent les femmes sur leurs visages avec deux «brèches » (kharqân) en guise d'yeux ${ }^{\mathbf{1 6}}$. Al-mubarqa'a est la shât (la brebis). Brebis ou chamelle ou animal rampant au plus bas de l'échelle animale, elle peut être débridée. D'où :

« Admonestez celles dont vous craignez l'infidélité (nushûz);

Reléguez-les dans des chambres à part et frappez-les ».

Il ne s'agit point d'infidélité, comme le veut la traduction, mais de nushûz. Tous les exégètes s'accordent pour dire que ce terme signifie le sentiment de supériorité, isti'lâ' 'alâ azwâjihinna (se sentir supérieures à leurs époux), comme l'écriront Tabarî et Râzî qui établissent une échelle dans cette taxinomie de la punition: d'abord, les réprimander, avant de les reléguer dans leurs chambres, avant de les frapper si elles persistent à désobéir. À quoi ? À Dieu et à leurs maris, répond Tabarî.

Au fur et à mesure qu'on avance dans les commentaires, on est frappé par le plaidoyer pour un art «quantitatif de la souffrance» (expression de M. Foucault). La punition est réglée (frapper avec un miswak, branche d'un arbre, ne pas frapper le visage car ce dernier est majma 'al-mahâsin, le lieu de la beauté). Mais, ce qui apparaît, à première vue, comme compassion s'avère cruelle affirmation d'un droit du pouvoir et de sa supériorité intrinsèque. L'homme, dans cette taxinomie du châtiment, adopte ce qu'il considère comme « la plus grande des humiliations » à savoir posséder la femme charnellement dans un mutisme voulu et absolu. Et Tabarî de continuer: «Persister à ne pas lui adresser la parole et la posséder, est très dur pour elle (wa dhâlika ashaddu 'alayha)». Ou encore, l'homme la délaisse et refuse de partager sa couche jusqu'à ce qu'elle revienne vers lui (soumise) et fasse ce qu'il désire (hattâ tarji'a ilâ mâ yuhibb), ou, écrit encore Tabari, « jusqu'à ce qu'elle respecte l'impératif divin de se soumettre à vos droits $\gg^{17}$ (il s'agit des droits des époux). Le respect de l'homme devient devoir divin et la loi divine se confond avec la loi de l'homme.

Trouver une technique pour y ajuster les punitions fera du corps féminin, dans cette 《sémiotechnique de la punition ${ }^{\mathbf{1 8}}$, le personnage principal. Soit il sera châtié, soit abandonné, délaissé comme corps érogène en souffrance, traversé par le désir et l'appel à l'autre qui ne répond pas, ou pris dans l'avilissement. Ainsi, ce qui paraît relever d'une «esthétique raisonnable» s'avère une violence inouïe. Le corps est pris dans les méandres d'un pouvoir qui «s'exalte et se renforce de ses manifestations physiques $»^{19}$. Le système punitif est à plala nuque.

16. La pièce d'étoffe qui protège la tête du cheval avec une partie articulée qui se rabat sur

17. Tabarî, Jâmi'al-bayân, Beyrouth, éd. Dâr al-fikr, 1988, tome5-6, p. 64.

18. Foucault, Surveiller, punir. Naissance de la prison, Gallimard, col. Tel, 1975, p.22

19. Foucault M., op. cit., p.69. 
cer dans une certaine économie politique qui assujettit le corps féminin et opère sur lui «une prise immédiate» autant physique que psychique.

Des exégètes des premiers siècles de l'islam jusqu'aux fuqahâ' (docteurs de la loi) d'aujourd'hui, le corps de la femme est soit productif (maternité), soit assujetti (et les deux à la fois). Le mari jugeant de l'âme de sa compagne a le droit de la punir, sur décret divin. À ce moment, châtier devient un droit souverain transformant ainsi le pouvoir en «surpouvoir» (expression de M. Foucault) car la justice risque de s'effacer au profit des aléas de l'arbitraire.

Dans le chapitre qui traite du châtiment réservé aux femmes, Tabarî relate cette histoire. Un homme frappa sa femme sur le visage et celle-ci vint chez le prophète demander justice. Ce dernier voulut la venger, mais l'Ange lui révéla le verset : «Ne te hâte pas dans la récitation, avant que sa Révélation ne soit achevée pour toi $»^{20}$. Or, ce verset, n'est nullement en lien avec les femmes, mais avec des réponses hâtives que le prophète aurait livrées aux Juifs qui cherchaient à tester la solidité de sa science. D'où la suite : « Dis : Mon Seigneur, augmente ma science $»^{21}$.

Lorsqu'il s'agit des femmes, les commentateurs se hâtent de mettre le divin du côté du principe masculin. Leur élocution au sujet du paradis et ce qui est promis aux hommes dans l'au-delà est, à ce titre, exemplaire. Le sexuel devient une orgie interminable et une jouissance masculine illimitée. Face au cortège interminable des houri, la virilité de l'homme, dépeinte comme absolue, compromet l'idée même du plaisir sexuel, puisque le sexe, dans cet imaginaire, reste constamment congestionné, douloureux, en perpétuelle possession, sans possibilité de décharge ${ }^{22}$. Suyûtî dépeint une verge qui ne se repose jamais faisant de l'homme du paradis le frère d'Ouranos. Si certains versets évoquent une certaine sensorialité primitive (fleuve de lait et de miel...) et la levée de tout interdit: «des échansons (jeunes éphèbes)», les exégètes, quant à eux, renforcent l'idée d'un plus de jouissance. Le sexe devient un fait discursif, investi par toute une trame de discours religieux, théologiques, juridiques... Il est stylisé, narré, objet premier des spéculations qui ne font que ... s'en occuper.

L'image paradisiaque d'une jouissance masculine à l'infini trouve son répondant dans une langue, d'une richesse sémantique extraordinaire, qui demeure néanmoins prisonnière d'une société encore gérée par une concep-

20. Cor. $20: 14$.

21. Cor. $20: 114$.

22. «Et bien, nous connaissons le modèle d'un organe douloureusement sensible, modifié en quelque façon sans être pour autant malade au sens habituel : c'est l'organe génital en état d'excitation», Cf. Freud S., «Pour introduire le narcissisme», in La vie sexuelle, PUF, 1969, p. 90. 
tion archaïque de la vie et de la société. Le mot vierge " 'adhrâ », à titre d'exemple, ne désigne que la jeune fille. Il ne se dit jamais au masculin, comme si l'homme était soustrait à tout devenir, à toute évolution et à toute historisation. On naît homme, on ne le devient pas. La lubricité interroge, ainsi, la place accordée aux femmes dans l'imaginaire musulman. La femme reste un corps, possédé quand l'homme le désire ou pris dans les fonctions domestiques d'icibas. Qu'en est-il de sa jouissance à elle? Comment sera-t-elle récompensée? Un voile sur sa sexualité de femme. Son destin se trouve ainsi scellé : obéissance ici-bas et chasteté dans l'au-delà. Ainsi en va-t-il des femmes.

\section{L’INQUIÉTANTE ÉTRANGETÉ D’UN VISAGE SEXE}

Mêlant facialité et monstruosité, Gorgô porte la mort dans les yeux. Elle pétrifie quiconque ose la regarder de face. Comment voir alors ce dont on ne peut soutenir la vue, le regarder sans courir le risque d'être pétrifié ? Grâce au bouclier d'Athéna, qui permet de voir Méduse non dans la réalité de sa personne mais en image, Persée pourra décapiter Gorgô sans la voir directement.

Décapiter, dira Freud, c'est castrer. À ce moment, «l'effroi devant la Méduse est donc effroi de la castration $»^{23}$. Et Ferenczi d'ajouter : «la tête de la Méduse comme le symbole effrayant de la région génitale féminine dont les caractéristiques ont été déplacées «du bas vers le haut ${ }^{24}$. «L'analyse des rêves, continue Ferenczi, et des associations m'a amené plusieurs fois à interpréter la tête de Méduse comme le symbole effrayant de la région génitale féminine ». La tête de Méduse se substitue à la figuration de l'organe génital féminin qui est l'organe génital de la mère.

Est-ce pour empêcher la pétrification de l'homme, que la femme doit voiler sa chevelure ? Â ce moment, le voile ne signifie pas uniquement la servitude de la femme sur la scène sociale, mais son assujettissement à un discours sur le corps féminin en lien avec ce qui demeure chez l'homme comme angoisse archaïque. Exemplaire est le récit que nous content les historiographes : la liquidation du Panthéon des déesses-mères de la période préislamique fut particulièrement difficile «et un envoyé du Prophète, chargé de mettre fin au règne d'une de ces trois déesses, dut s'y reprendre à plusieurs fois à cause de l'aspect physique monstrueux et menaçant de cette dernière. C'était « une femme abyssine, aux cheveux ébouriffés, posant les mains sur les épaules et grinçant des dents $»^{25}$.

23. Freud, «La tête de Méduse », in Résultats, idées, problèmes, II, PUF, 1981, p. 49.

24. Ferenczi, Euvres complètes, Psychanalyse 3, Payot, 1974, p. 200.

25. Françoise Couchard, Le fantasme de séduction dans la culture musulmane, PUF, 1994, p. 64. 
Or, nous savons que les Grées forment un inquiétant trio : vieilles sorcières juvéniles avec une seule dent et un seul œil pour trois transmis continûment d'un visage sur un autre. Il est sans cesse ouvert, toujours aux aguets, équivalent aux cent yeux d'Argos qui permettent de regarder de tous les côtés.

Cheveux ébouriffés, noire et grinçant des dents, menaçant de dévoration, le regard terrifiant. Monstruosité, oralité cannibalique, image terrifiante et grotesque, n'est-ce pas l'image même de l'archaïque? Aussi cette image ne se réduit-elle pas aux «lointains de l'horizon civilisé mais ce qui, à tout moment et en tout lieu, arrache l'homme à sa vie et à lui-même (...) pour le projeter vers le bas, dans la confusion et l'horreur du chaos $»^{26}$.

«Les yeux ne perçoivent pas seulement les modifications du monde extérieur, importantes pour la conservation de la vie, écrit Freud, mais également les propriétés des objets par lesquelles ceux-ci sont élevés au rang d'objets du choix d'amour, leur attraits $»^{27}$. Aussi l'organe de la vue devient-il le lieu de l'angoisse ${ }^{28}$. Les mythes, les rêves et les fantasmes nous ont appris que l'angoisse de perdre la vue est un substitut de l'angoisse de castration. Et Freud de rattacher angoisse de castration et inquiétante étrangeté (Unheimlich). Or, «Heimich est un mot dont la signification évolue en direction d'une ambivalence, jusqu'à ce qu'il finisse par coïncider avec son contraire, unheimlich », à savoir cette «variété particulière de l'effrayant qui remonte au depuis longtemps connu, depuis longtemps familier $»^{29}$. Le familier qui aurait dû rester caché. Serait donc unhemlich ce qui aurait dû rester secret, dans l'ombre mais qui en est sorti. À ce moment, la vue est en prise avec la résonance angoissante du fantasme de la castration.

Puisque l'œil n'est pas seulement un organe d'auto-conservation, mais porte la puissance de la pulsion érotique, pour éviter la loi du Talion, il vaut mieux voiler que perdre la vue ${ }^{30}$.

26. J.-P. Vernant, La mort dans les yeux, Hachette, 1975, p. 30.

27. Freud «Le trouble psychogène de la vision », in Névrose, psychose et perversion, PUF, 1981, p. 171.

28. P. Fédida, «L'angoisse aux yeux », in Par où commence le corps humain, PUF, 2000, p. 61.

29. Freud S., "L'inquiétante étrangeté », in L'inquiétante étrangeté et autres essais, Gallimard, Folio/Essais p. 223 et p. 215.

30. «Si la pulsion sexuelle partielle qui se sert du regard, la scoptophilie sexuelle, a attiré sur elle en raison de ses prétentions excessives la contre-offensive des pulsions du moi, de sorte que les représentations dans lesquelles s'expriment ses aspirations succombent au refoulement et sont écartées de l'accession à la conscience est de ce fait totalement perturbée. » (Freud, «Le trouble psychogène de la vision », in op.cit., p. 171 et Freud dira un peu plus loin «Puisque tu as voulu mésuser de ton organe visuel en t'en servant pour un malin plaisir sensuel, ce n'est que justice si tu ne vois plus rien du tout. » p. 172. 


\section{FEMME, LE PLUS NOBLE DES MOTS}

Le voile et la féminité sont un leitmotiv de la pensée mystique. À l'encontre de la vision théologique, le paradis que prône le texte mystique est celui des intelligences où l'âme pourra éprouver l'union sans voile ${ }^{31}$. Et dans un bouleversement des frontières : humain/divin, la mystique parlera de la possibilité d'une vision directe, bouleversante et jouissive du divin. C'est en termes de vision qu'est décrite la fusion avec l'Un. « Nul besoin qu'Il soit invité, Il est mon convive à jamais » ou «Ton image qui passait est devenue l'invitée de mes yeux. Elle choisit mes yeux pour patrie ${ }^{32}$. Et «deviens regard, deviens regard, deviens regard $»^{33}$.

Si l'une des paraboles de Mantic uttair ${ }^{34}$, consiste à utiliser le motif du miroir pour atténuer la vision de la splendeur divine, le véritable mystique est celui qui accepte de se brûler non pas pour l'objet de son désir, mais dans l'objet de son désir. Consumé à l'instar du papillon qui s'unit à la bougie, il emporte le secret de cette union avec lui et demeure ainsi enveloppé dans son mystère... à tout jamais.

Toutefois, si pour certains mystiques, tel al-Ansârî, le voile peut chuter ${ }^{35}$, pour Ibn Arabi, à certains moments de ses constructions, le voile ne chute jamais. Car les mots sont des voiles, les lettres sont également un voile. Quant au moi (ou l'ego), il est akthafu al-hujub al-hissiyya (le plus épais des voiles sensibles $)^{36}$.

Et c'est dans cette approche du miroir et de l'image qu'apparaît la femme. Elle prête même ses traits au divin. Il est Layla, belle et bien-aimée, une convive à jamais. Au sein, de ce corpus, ce n'est pas l'image du fils qui réfléchit le divin, mais la femme. Et pour Ibn Arabi, qui écrit: «la féminité est ce qui circule dans le monde », la contemplation de Dieu dans la femme est la plus parfaite ${ }^{37}$.

31. Nadia Tazi, « D’un paradis obscur », in Cliniques méditerranéennes, $\mathrm{n}^{\circ} 73,2006$, pp. 97-114.

32. Rûmî, Rubâ'iyyât, trad. E. Meyerovitch, et J. Mortazavi, éd. Albin Michel, 1987, p. 184.

33. Cité par Eva Meyérovitch, Poésie et mystique en islam, p. 111.

34. F.-D. Attâr, Mantic uttair (Le langage des oiseaux), trad. M. Garcin de Tassy, éd. Aujourd'hui, 1863.

35. L'équivalent de l'ego.

36. Cf. H. Abdelouahed, «Des noms qui regardent», in Cliniques méditerranéennes, $\mathrm{n}^{\circ}$ 51/52, 1996, pp. 145-155.

37. Ibn Arabi, Fusûs al-hikam, Beyrouth, éd. Dâr al-Kitâb al- 'arabî, 1946, p. 217 ; La sagesse des prophètes, Albin Michel, 1974, p. 201. 


\section{NÉCESSITÉ D’UNE CONSTRUCTION}

"C'est le rapport de chaque discours à la mort qui le rend possible », écrit M. de Certeau ${ }^{38}$. Or, nos théologiens, non seulement dénient, au nom de l'accrochage à la tradition, la charte des Droits de l'homme et les principes civiques parce que nés en Occident, mais opèrent, au sein de l'immense corpus arabo-musulman, une dichotomie confinant au silence mystique, poésie, 'ilm al-kalâm (comme scolastique de l'islam), philosophie... Condamnant ainsi la pensée, ils poussent à une régression jamais égalée dans l'histoire des civilisations. Brandissant à l'orée du troisième millénaire sans cesse l'étendard de l'identité, ils s'opposent à un travail d'écriture au présent et condamnent la succession des générations ainsi que toute possibilité d'ouverture sur un avenir commun avec les autres. Or, le travail sur la source nécessite la béance d'un écart. Et l'identité est une construction, jamais achevée, toujours à venir, transformée par l'épreuve de l'étranger. Nos fuqahâ', à l'orée du troisième millénaire, ne cessent de multiplier les fatwa sur le démon qui ne cesse de harceler l'humain. Or, le démon n'est que la projection de cette part obscure de nous-mêmes. Et cette violence contre les femmes en dit long sur la non-acceptation de cet Autre en soi, le refus de la part féminine, opaque et énigmatique, que les hommes portent en eux-mêmes. La violence signe l'échec de cette capacité de se transcender soi-même " afin d'assurer son unité véritable » (selon l'expression de René Nelli).

« Le cœur de l'amant est tout entier roseraie », chantait Rûmî.

Pour nos fuqahâ', l'amour a déserté le monde, de même que toute capacité de sublimation et de métaphorisation. C'est comme si, désormais, le monde entier n'était que ronces.

Houria ABDELOUAHED

255 rue d’Épinay 95360 Montmagny

houriya_abdelouahed@yahoo.fr 


\section{BIBLIOGRAPHIE}

Abdelouahed H., « Des noms qui regardent », in Cliniques méditerranéennes, $\mathrm{n}^{\circ}$ 51/52, 1996, pp. 145-155.

Abdelouahed H. \& Adonis, Le Dîwân de la poésie arabe classique, Gallimard /Poésie, 2008.

Attâr F.-D., Mantic uttair (Le langage des oiseaux), trad. M. Garcin de Tassy, éd. Aujourd'hui, 1863.

Certeau M. de, L'écriture de l'histoire, Gallimard, 1975, Col. Folio, p.74

Couchard F., Le fantasme de séduction dans la culture musulmane, PUF, 1994.

Fédida P., «L'angoisse aux yeux », in Par où commence le corps humain, PUF, 2000.

Ferenczi S., Euvres complètes, Psychanalyse 3, Payot, 1974, p. 200.

Foucault, Surveiller, punir. Naissance de la prison, Gallimard, col. Tel, 1975.

Freud S., «Le trouble psychogène de la vision » (1910), in Névrose, psychose et perversion, PUF, 1983.

Freud S., « Pour introduire le narcissisme » (1914), in La vie sexuelle, PUF, 1969.

Freud S., «L'inquiétante étrangeté » (1919), in L'inquiétante étrangeté et autres essais, Gallimard, 1985, Folio/Essais.

Freud S., «La tête de Méduse » (1925), in Résultats, idées, problèmes, II, PUF, 1981.

Ibn Arabi, Fusûs al-hikam, Beyrouth, éd. Dâr al-Kitâb al- 'arab̂̂, 1946 ; La sagesse des prophètes (trad. Titus Burckardt), Albin Michel, 1974.

Legendre P., Dieu au miroir, Fayard, 1994.

Qaradawi Y., Le licite et l'illicite en islam., réédition Dâr-al qalam, en 2004.

Râzî, At-Tafsîr al-kabîr, tome 9-10, éd. Dâr al-kutub al- 'ilmiyya, Beyrouth.

Rûmî, Rubâ'iyyât, trad. E. Meyerovitch, et J. Mortazavi, éd. Albin Michel, 1987, p. 184.

Tabarî, Jâmi'al-bayân, tome5-6, Beyrouth, éd. Dâr al-fikr, 1988.

Tabâtibâ'î, Al-Mîzân fì tafsîr al-Qur'ân, tome 4, Beyrouth, éd. Manshûrât mu'assassat ala'lâ.

Tazi N., « D’un paradis obscur », in Cliniques méditerranéennes, $\mathrm{n}^{\circ}$ 73, 2006, pp. 97-114.

Vernant J.-P., La mort dans les yeux, Hachette, 1975.

Vernant J.-P., L'individu, la mort, l'amour, Paris, Gallimard, 1989.

Zweig S., Le joueur d'échecs, Stock, 1990. 


\section{Houria Abdelouahed - Ce voile qui cache la forêt}

Résumé : Le nouveau malaise dans la culture arabo-musulmane, à travers le problème du hijâb (traduit par « voile») pivote autour d'un entrelacs entre voir et être vu.

Si le Texte prône une vision conservatrice de la femme, les différents commentaires des exégètes, au Moyen Âge et aujourd'hui, font miroiter des angoisses archaïques face au corps féminin. Le visage cesse d'être le miroir - où l'autre voit le reflet dédoublé de lui-même -, pour devenir l'unheimlich effrayant. D'où la nécessité de faire le lien entre l'inquiétante étrangeté et la tête de Méduse, la plus mortelle des trois Grées qui revient de façon extraordinaire dans les écrits des historiographes autour des déesses-mères préislamiques.

Mots-clés : Voile - Inquiétante étrangeté - Femme - Mystique - Identité Construction.

\section{Houria Abdelouahed - We Can't See the Tree for the Veil}

Summary : The recent unease in the Arab and Muslim world highlighted by the question of the hijâb (translated as 'veil') revolves around the articulation of being seen and seeing. While religious texts advocate a conservative vision of woman, commentaries on these texts by exegetes from the Middle Ages to current times, reflect the archaic anxieties aroused by the female body in this light. A veiled face ceases to be the mirror in which the other perceives his own reflection and becomes an expression of the Uncanny in its most terrifying form. Hence the need to link the Uncanny with Medusa's head. Medusa was the most deadly of the three gorgons and makes an astonishing appearance in much of the writing of historiographs on pre-Islamic mother-goddesses.

Key-words : Veil - the Uncanny - Woman - Mystic - Identity - Construction. 